\title{
Improving Estimation Performance in Networked Control Systems Applying the Send-on-delta Transmission Method
}

\author{
Vinh Hao Nguyen and Young Soo Suh * \\ Department of Electrical Engineering, University of Ulsan, Namgu, Ulsan 680-749, Korea \\ E-mail: vinhhao@hcmut.edu.vn.
}

* Author to whom correspondence should be addressed. Email: suh@ieee.org

Received: 4 September 2007 / Accepted: 2 October 2007 / Published: 5 October 2007

\begin{abstract}
This paper is concerned with improving performance of a state estimation problem over a network in which a send-on-delta (SOD) transmission method is used. The SOD method requires that a sensor node transmit data to the estimator node only if its measurement value changes more than a given specified $\delta$ value. This method has been explored and applied by researchers because of its efficiency in the network bandwidth improvement. However, when this method is used, it is not ensured that the estimator node receives data from the sensor nodes regularly at every estimation period. Therefore, we propose a method to reduce estimation error in case of no sensor data reception. When the estimator node does not receive data from the sensor node, the sensor value is known to be in a $\left(-\delta_{i},+\delta_{i}\right)$ interval from the last transmitted sensor value. This implicit information has been used to improve estimation performance in previous studies. The main contribution of this paper is to propose an algorithm, where the sensor value interval is reduced to $\left(-\delta_{i} / 2,+\delta_{i} / 2\right)$ in certain situations. Thus, the proposed algorithm improves the overall estimation performance without any changes in the send-on-delta algorithms of the sensor nodes. Through numerical simulations, we demonstrate the feasibility and the usefulness of the proposed method.
\end{abstract}

Keywords: Kalman filter, Estimation, Sensor networks, Send-on-delta. 


\section{Introduction}

Recently, interest in the study of networked control system (NCS) and sensor networks has increased widely due to its low cost, high flexibility, simple installation and maintenance [1]. In such systems, a center station has the task of receiving, processing and sending data to the slave nodes such as sensor nodes, actuator nodes, etc. over a serial network. It is easy to add or remove slave nodes in the network without changing the system structure much. Aside from the advantages as mentioned above, however, there are several problems affecting to the system such as bandwidth, networkinduced delay, packet loss rate.

One of the most interesting problems is how to reduce network bandwidth when there are many nodes on the network. This can be achieved by reducing either data packet size or data packet transmission rate. In [2], an adjustable deadband was defined on each node to reduce network traffic. The node does not broadcast a new message if its signal is within the deadband. In [3], estimators were used at each sensor node instead. When the estimated value deviates from the actual output by more than a prespecified tolerance, the actual sensor data are transmitted. To overcome the limited network bandwidth, transmission data size reduction using a special encoder-decoder was considered in [4]. Another method for reduction of data transmission rate called send-on-delta (SOD) transmission was explored in [5-8]. This method requires that a sensor node transmit data to the estimator node only if its measurement value changes more than a given specified $\delta$ value. By adjusting the $\delta$ value at each sensor node, data transmission rate is reduced so that the network bandwidth is increased and can be used for other traffic.

The purpose of this paper is to extend our work on the modified Kalman filter employing a SOD transmission method [5], where the states are periodically estimated by the estimator node regardless of whether the sensor nodes transmit data or not. A challenging issue is how the estimator node determines the measurement value at a sensor node if it does not send data. If this problem is well solved, the estimation performance will be significantly improved. In this paper, we examine and evaluate the measurement output value as well as measurement noise arising when a sensor node does not transmit data. Then, a computed output with the new measurement value and new noise covariance is compensated to the system in order to reduce estimation error. Through simulations, we show that the proposed method gives better estimation performance than that of [5].

\section{Sensor Output Evaluation}

Consider a networked control system illustrated as in Figure 1, where the linear continuous-time system is described as:

$$
\begin{aligned}
\dot{x}(t) & =A x(t)+B u(t)+w(t) \\
y(t) & =C x(t)+v(t)
\end{aligned}
$$

where $x \in R^{n}$ is the state of the plant, $u$ is the deterministic input signal, $y \in R^{p}$ is the measurement output which is sent to the estimator node by the sensor nodes. $w(t)$ is the process noise with covariance $Q$, and $v(t)$ is the measurement noise with covariance $R . w(t)$ and $v(t)$ are uncorrelated, zero mean white Gaussian random processes. 
The following assumptions are made on the data transmission over network:

1. Measurement outputs $y_{i}(1 \leq i \leq p)$ are sampled at period $T$, but their data are only transmitted to the estimator node when the difference between the current value and the previously transmitted one is greater than $\delta_{i}$.

2. For simplicity in problem formulation, any transmission delay from the sensor nodes to the estimator node is ignored.

Figure 1. Configuration of a networked control system

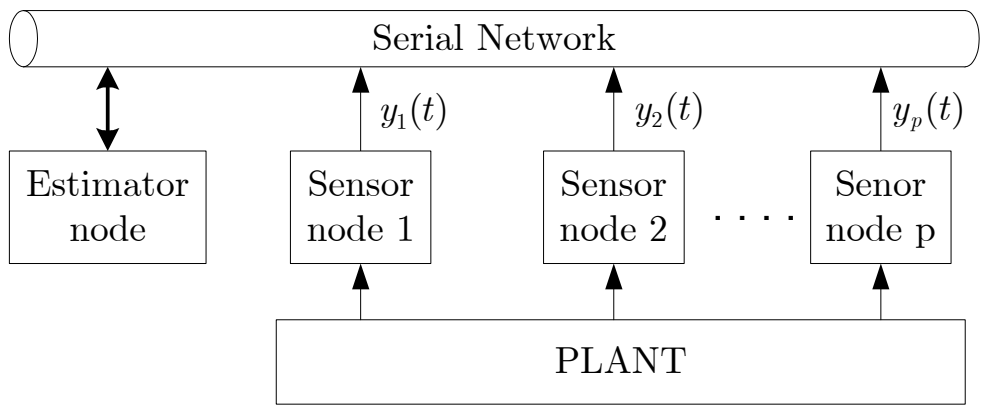

The estimator node estimates the states of the plant regularly at period $T$, regardless of whether or not any sensor data arrive. If the $i$-th sensor data do not arrive, the estimator node knows that the current value of $i$-th sensor output has not changed more than the range $\left(-\delta_{i},+\delta_{i}\right)$, compared with the last arriving one. This implicit information is used to estimate the current states in case $i$-th sensor data do not arrive.

Let the last received value of $i$-th sensor output be $y_{\text {last }, i}$ at time $t_{\text {last }, i}$. If there is no sensor data received for $t>t_{\text {last }, i}$, the estimator node considers that the measurement value of the $i$-th sensor output $y_{i}(t)$ is still equal to $y_{\text {last }, i}$ but the measurement noise is increased from $v_{i}(t)$ to $v_{n, i}(t)=v_{i}(t)+\Delta_{i}\left(t, t_{\text {last }, i}\right)$, where $\Delta_{i}\left(t, t_{\text {last }, i}\right)$ is defined [5]:

$$
\Delta_{i}\left(t, t_{\text {last }, i}\right)=y_{\text {last }, i}-y_{i}(t),\left|\Delta_{i}\left(t, t_{\text {last }, i}\right)\right| \leq \delta_{i}
$$

In [5], it was assumed that $\Delta_{i}\left(t, t_{\text {last }, i}\right)$ had a uniform distribution with zero mean and a variance $\delta_{i}^{2} / 3$. However, this assumption is incorrect in some cases where that measurement noise $R_{(i, i)}$ is smaller than $\delta_{i}^{2}$, where $R_{(i, i)}$ is the $(i, i)$-th element of $R$. To illustrate this, assume that there is no measurement noise (i.e., $R=0$ ). Then $0 \leq \Delta_{i}\left(t, t_{\text {last }, i}\right)<\delta_{i}$ if $C x(t)$ is increasing and $-\delta_{i}<\Delta_{i}\left(t, t_{\text {last }, i}\right) \leq 0$ if $C x(t)$ is decreasing. In this case, a reasonable assumption is that the mean of $\Delta_{i}\left(t, t_{\text {last }, i}\right)$ is $\delta_{i} / 2$ if $C x(t)$ is increasing and $-\delta_{i} / 2$ if $C x(t)$ is decreasing. This trend is preserved as long as the measurement covariance $R \ll \delta^{2}$. When the measurement covariance is large, then $\Delta_{i}\left(t, t_{\text {last }, i}\right)$ in [5] is more likely affected by the zero-mean measurement noise $v(t)$; thus zero mean assumption is a more valid assumption.

To demonstrate this argument, consider an example of the output response of an $2^{\text {nd }}$ order system with step input shown in Figure 2. The top graph is the output signal without noise $C x(t)$ and the measurement output $y(t)$. In this example, $y(t)$ is sampled by the send-on-delta scheme with $\delta=0.1$. The new measurement noise $v_{n}(t)$ is shown in the middle and bottom graphs for two cases: $R>\delta^{2}$ and $R \ll \delta^{2}$, respectively. Obviously, the waveform of $v_{n}(t)$ is rather different in two cases: 
It is a uniform distribution with zero mean in the first case, but with non zero mean in the second case. For example, $v_{n}(t)$ has a positive mean value in time range $(0,10 s)$ but a negative mean value in $(10,20 s)$.

Therefore, the assumption in [5] is only correct in the case $R>\delta^{2}$. Furthermore, in realistic systems, measurement noise is normally small, while $\delta$ is set to a greater value to reduce data transmission rate. For some types of digital sensor such as encoders, measurement noise is even equal to zero. This means that the second case (non-zero mean case) happens more usually than the first case in the real applications. We propose an estimation algorithm which exploits this non-zero mean case in order to improve the estimation performance.

Now we compute the mean value and variance of $v_{n, i}(t)$. Notice that the new measurement noise $v_{n, i}(t)$ only exists when the estimator node does not receive data from the $i$-th sensor node at instant $t$. Otherwise, measurement noise is still $v_{i}(t)$.

+ If $R_{(i, i)}>\delta_{i}^{2}:$

$$
\begin{gathered}
E\left\{v_{n, i}(t)\right\}=0 \\
\operatorname{Var}\left\{v_{n, i}(t)\right\}=R_{(i, i)}+\delta_{i}^{2} / 3 \\
+ \text { If } R_{(i, i)} \leq \delta_{i}^{2}: \\
E\left\{v_{n, i}(t)\right\}=\delta_{i} / 2\left(\text { or }-\delta_{i} / 2\right) \\
\operatorname{Var}\left\{v_{n, i}(t)\right\}=R_{(i, i)}+\delta_{i}^{2} / 12
\end{gathered}
$$

We see that variance of $v_{n, i}(t)$ in the second case (4) is smaller than in (3) which was used in [5].

Figure 2. Effect of $R$ and $\delta$ on measurement noise $v_{n}(t)$.
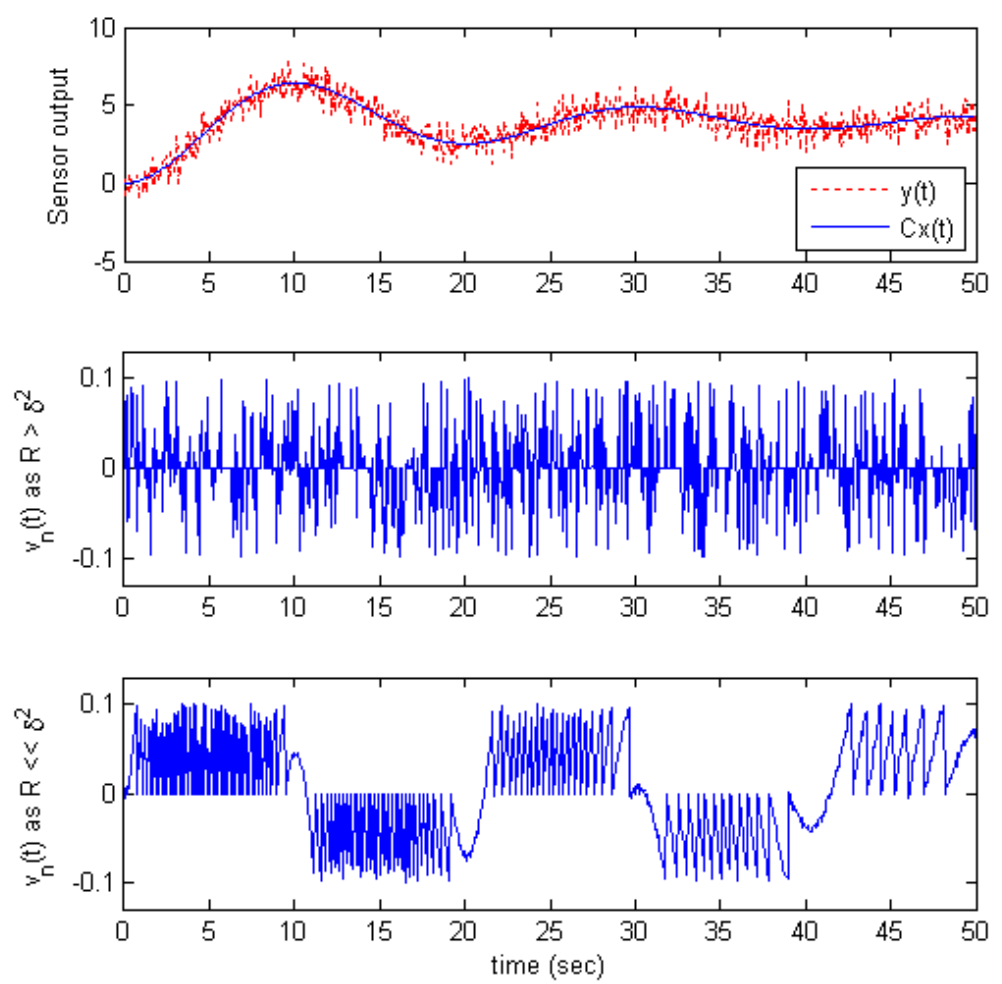


\section{State estimation with the SOD transmission method}

In this section, we propose a method to adaptively use (3) or (4) in the modified Kalman filter for the state estimation problem. The main issue here is when we should use (4) instead of (3)? And if (4) is used, how then to determine when the mean value of $v_{n, i}(t)$ is positive or negative?

Firstly, we compute the mean value of $v_{n, i}(t)$ when (4) is used. Let two latest consecutive $i$-th sensor output values received at the estimator node be $y_{\text {last }-1, i}, y_{\text {last }, i}$ at time $t_{\text {last }-1, i}, t_{\text {last }, i}$, respectively. Derivative of $y_{i}$ at time $t_{\text {last }, i}$ is approximately calculated as follows:

$$
y_{i}^{\prime}\left(t_{\text {last }, i}\right) \approx \frac{\hat{y}_{\text {last }, i}-\hat{y}_{\text {last }-1, i}}{t_{\text {last }, i}-t_{\text {last }-1, i}}=\frac{\hat{y}_{\text {last }, i}-\hat{y}_{\text {last }-1, i}}{k_{i} T}
$$

where $\hat{y}_{\text {last }, i}$ is the estimated value of $y_{i}(t)$ at instant $t_{\text {last }, i}$, and $k_{i}$ is number of sampling times. We use $\hat{y}_{\text {last }, i}$ instead of $y_{\text {last }, i}$ to reduce measurement noise effects. If the $i$-th sensor node does not send data at instant $t>t_{\text {last }, i}$, it is more likely that the output value $y_{i}(t)$ will satisfy:

$$
\hat{y}_{\text {last }-1, i}<\hat{y}_{\text {last }, i}<y_{i}(t)
$$

or

$$
y_{i}(t)<\hat{y}_{\text {last }, i}<\hat{y}_{\text {last }-1, i}
$$

Therefore, mean value of the new measurement noise $v_{n, i}(t)$ is computed:

$$
E\left\{v_{n, i}(t)\right\} \triangleq \operatorname{sign}\left(y_{i}^{\prime}\left(t_{\text {last }, i}\right)\right) * \delta_{i} / 2
$$

Once $E\left\{v_{n, i}(t)\right\}$ is obtained in (6), to satisfy zero mean noise condition in the Kalman filter, we just add this value to the measurement value $y_{\text {last }, i}$.

Now we investigate the system response to determine when (3) or (4) is chosen to the filter. The basic principle is that if it is certain that $y_{i}(t)$ is increasing or decreasing, we should use (4). And if it is not certain, we should use (3). This decision is made based on the absolute value of $y_{i}^{\prime}(t)$ as follows:

$$
\text { Selector }=\left\{\begin{array}{l}
(3), \text { if }\left|y_{i}^{\prime}\left(t_{\text {last }, i}\right)\right| \leq \varepsilon_{i} \\
(4), \text { otherwise }
\end{array}\right.
$$

where $\varepsilon_{i}>0$ is a sufficiently small threshold. If $\left|y_{i}^{\prime}\left(t_{\text {last }, i}\right)\right|$ is small, it is difficult to draw a meaningful conclusion about whether $y_{i}(t)$ is increasing or decreasing, so in that case, we use (3). Otherwise, we could be fairly certain about whether $y_{i}(t)$ is increasing or decreasing; in that case, we use (4). Some remarks about the parameter $\varepsilon_{i}$ are warranted. If $\varepsilon_{i}=\infty$, (3) is always used in the filter as in [5]. Therefore, in case $R_{(i, i)}>\delta_{i}{ }^{2}$, the proposed filter will become to the filter in [5] if setting $\varepsilon_{i}=\infty$. It is very flexible to switch the filter to [5] or to the the proposed filter so that estimation performance is improved in both case $R>\delta^{2}$ and $R \ll \delta^{2}$.

A modified Kalman filter for state estimation at step $k$, where there is a change in the measurement update part of the discrete Kalman filter algorithm, is given as in the Fig.3 when both (3) and (4) are used in case $R \ll \delta^{2}$. Basic principle of Kalman filters could be found in [10,11]. In Fig.3, we use the discretized plant model is sampled at period $T$ :

$$
A_{d}=e^{A T}, B_{d}=\int_{0}^{T} e^{A r} B d r
$$


$Q_{d}$ is the process noise covariance of the discretized plant:

$$
Q_{d}=\int_{0}^{T} e^{A r} Q e^{A^{\prime} r} d r
$$

$y_{\text {last }}$ is the vector of $p$ last received sensor values:

$$
y_{\text {last }}=\left[\begin{array}{llll}
y_{\text {last }, 1} & y_{\text {last }, 2} & \ldots & y_{\text {last }, p}
\end{array}\right]^{\prime},
$$

and $m$ is the vector of $p$ mean values:

$$
m=\left[\begin{array}{llll}
m_{1} & m_{2} & \ldots & m_{p}
\end{array}\right]^{\prime} .
$$

Figure 3. Structure of the modified Kalman filter loop

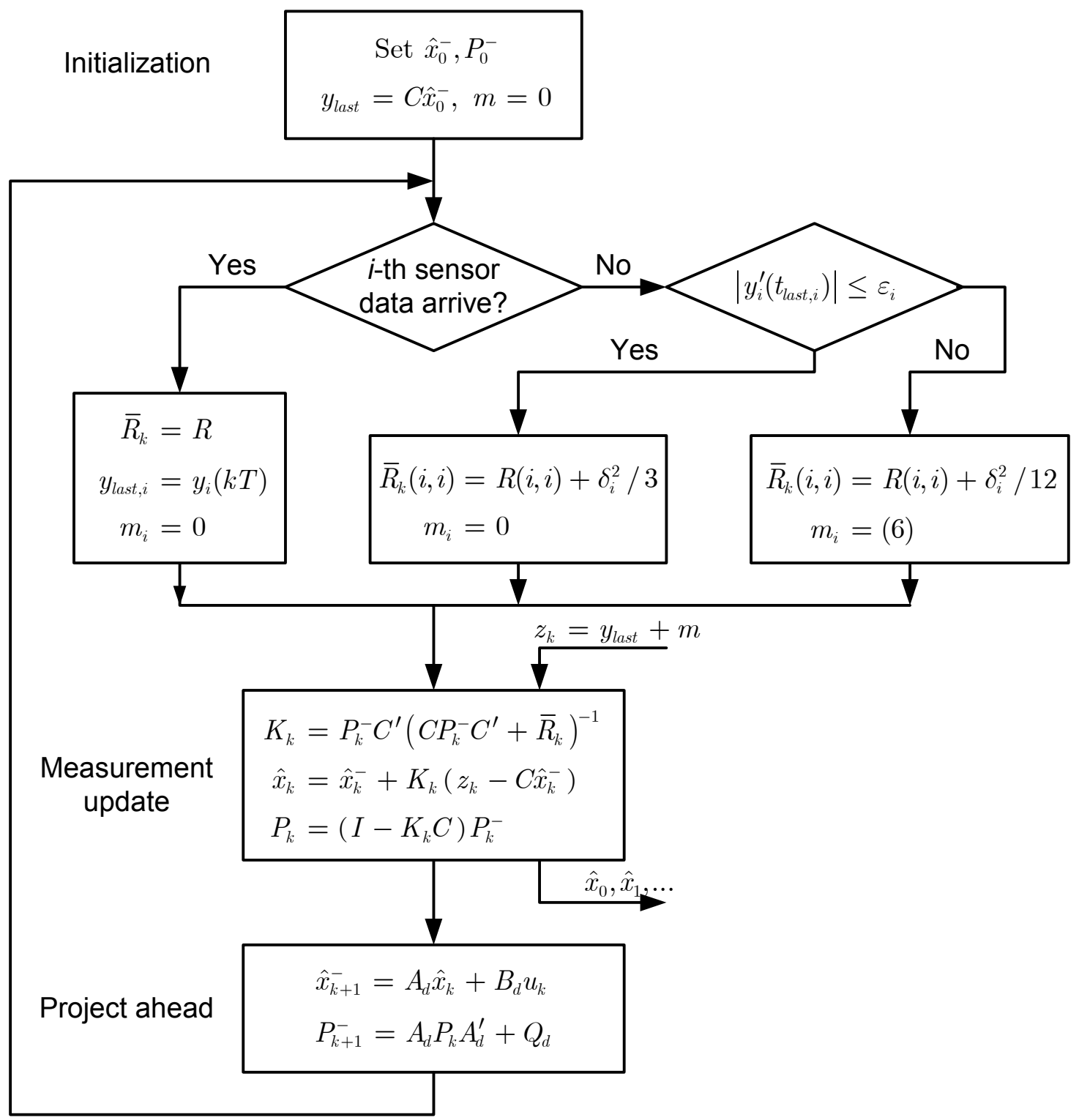


In the modified filter above, vector $m$ presents mean value of $v_{n}(t)$ for all $p$ measurement outputs. In case the filter uses (4) for estimation, $m$ becomes non-zero. In order to satisfy zero mean noise condition, we add $m$ to $y_{\text {last }}$ and consider $\left(y_{\text {last }}+m\right)$ as the measurement values.

The $\varepsilon_{i}$ thresholds determine when the filter uses (4) instead of (3). The smaller $\varepsilon_{i}$ is, the more the filter relies on (4). In practical systems, $\varepsilon_{i}$ thresholds could be determined by monitoring derivative of the sensor output $y_{i}^{\prime}(t)$.

It is difficult to derive an explicit expression of performance improvement. However, we could say that the error covariance $P_{k}$ becomes smaller when (4) is used more often. This is because smaller $\bar{R}$ value in the Kalman filter results in smaller $P_{k}$ value. We note that (4) is more often used if $y(t)$ is either monotonically increasing or decreasing.

\section{Simulation}

To verify the proposed filter, we consider an example of the step response of a second-order system where the output is sampled by the SOD method:

$$
\begin{aligned}
& \dot{x}(t)=\left[\begin{array}{cc}
0 & 1 \\
-1 / a & -b / a
\end{array}\right] x(t)+\left[\begin{array}{c}
0 \\
M / a
\end{array}\right] u(t)+w(t) \\
& y(t)=\left[\begin{array}{ll}
1 & 0
\end{array}\right] x(t)+v(t) \\
& Q=10^{-4}, R=10^{-4}, T=10 \mathrm{~ms}
\end{aligned}
$$

The system parameters are given in 3 following cases for performance evaluation:

1. $M=30, \mathrm{a}=10, \mathrm{~b}=10$ : overdamped system

2. $M=30, \mathrm{a}=5, \mathrm{~b}=1 \quad:$ underdamped system

3. $M=30, \mathrm{a}=5, \mathrm{~b}=0 \quad$ : undamped system

The simulation process is implemented for 50 seconds, and $\varepsilon=0.001$ for all cases. In each case, we use 3 filters for performance comparison: the filter using (3) only, the filter using (4) only, and the proposed filter. Estimation error is evaluated by the criterion:

$$
e=\left(\frac{1}{N} \sum_{k=1}^{N}\left(x_{1}-\hat{x}_{1}\right)^{2}\right)^{1 / 2}
$$

where $x_{1}$ is the reference state, $N=5000$.

Estimation errors for different $\delta$ values in 3 cases, where the condition $R \ll \delta^{2}$ is satisfied, are given in Table 1, 2, and 3. We see that estimation performance of the proposed filter is the best and significantly improved in comparison with the filter using (3). For example, in the case of $\delta=0.2$, estimation error of the proposed filter is reduced $36.75 \%$ compared to the filter using (3) in case 1 , reduced $0.88 \%$ in case 2 , and reduced $7.49 \%$ in case 3 .

The results also show that the filter using (4) is better than the filter using (3) in case 1, but worse in case 2 and case 3. In theory, the filter using (4) must be always better than the filter using (3). This happens because there exists a delay between the sensor output signal $y^{\prime}(t)$ and the computed value $y^{\prime}\left(t_{\text {last }}\right)$ in (5). Therefore, if $y^{\prime}(t)$ changes sign while condition $\left|y(t)-y\left(t_{\text {last }}\right)\right|<\delta$ still holds, the estimator keeps using the old value $y^{\prime}\left(t_{\text {last }}\right)$ whose sign is incorrect compared with $y^{\prime}(t)$. This makes 
(6) incorrect, and leads to increase of estimation error. As we see in the Fig.5 and Fig.6, the estimation error of the filter using (4) becomes large at time $y^{\prime}(t)$ changes the sign.

The proposed filter overcomes this situation by applying the algorithm (7) in which the filter uses (3) at time $y^{\prime}(t)$ changes sign and uses (4) at other time. The solid line on the proposed filter graphs in Fig.4, Fig.5, and Fig.6 presents the sign of Selector (7). That is, Selector $>0$ denotes that the filter uses (4) and Selector $<0$ denotes that the filter uses (3).

Table 1. Estimation error with different $\delta$ values in case 1.

\begin{tabular}{|c|c|c|c|c|}
\hline & $\delta=0.05$ & $\delta=0.1$ & $\delta=0.15$ & $\delta=0.2$ \\
\hline \hline$n$ & 454 & 268 & 185 & 141 \\
\hline Filter using (3) & $2.5643 \mathrm{e}-004$ & $3.2061 \mathrm{e}-004$ & $4.6965 \mathrm{e}-004$ & $5.6528 \mathrm{e}-004$ \\
\hline Filter using (4) & $1.9581 \mathrm{e}-004$ & $2.0124 \mathrm{e}-004$ & $3.0587 \mathrm{e}-004$ & $3.5756 \mathrm{e}-004$ \\
\hline Proposed filter & $1.9581 \mathrm{e}-004$ & $2.0123 \mathrm{e}-004$ & $3.0588 \mathrm{e}-004$ & $3.5755 \mathrm{e}-004$ \\
\hline
\end{tabular}

$n:$ number of sensor data transmissions

Table 2. Estimation error with different $\delta$ values in case 2 .

\begin{tabular}{|c|c|c|c|c|}
\hline & $\delta=0.05$ & $\delta=0.1$ & $\delta=0.15$ & $\delta=0.2$ \\
\hline$n$ & 1168 & 657 & 484 & 370 \\
\hline Filter using (3) & $0.9644 \mathrm{e}-004$ & $2.7051 \mathrm{e}-004$ & $4.1805 \mathrm{e}-004$ & $5.5656 \mathrm{e}-004$ \\
\hline Filter using (4) & $1.7065 \mathrm{e}-004$ & $2.9365 \mathrm{e}-004$ & $4.6972 \mathrm{e}-004$ & $5.5783 \mathrm{e}-004$ \\
\hline Proposed filter & $0.9468 \mathrm{e}-004$ & $2.6787 \mathrm{e}-004$ & $4.1551 \mathrm{e}-004$ & $5.5166 \mathrm{e}-004$ \\
\hline
\end{tabular}

Table 3. Estimation error with different $\delta$ values in case 3 .

\begin{tabular}{|c|c|c|c|c|}
\hline & $\delta=0.05$ & $\delta=0.1$ & $\delta=0.15$ & $\delta=0.2$ \\
\hline \hline$n$ & 4055 & 3091 & 1939 & 1700 \\
\hline Filter using (3) & $4.1432 \mathrm{e}-005$ & $5.1109 \mathrm{e}-005$ & $4.1805 \mathrm{e}-005$ & $6.5436 \mathrm{e}-005$ \\
\hline Filter using (4) & $4.4961 \mathrm{e}-005$ & $6.6618 \mathrm{e}-005$ & $4.6972 \mathrm{e}-005$ & $8.2118 \mathrm{e}-005$ \\
\hline Proposed filter & $3.9019 \mathrm{e}-005$ & $4.6125 \mathrm{e}-005$ & $4.1751 \mathrm{e}-005$ & $6.0537 \mathrm{e}-005$ \\
\hline
\end{tabular}


Figure 4. Estimation error of 3 filters $(\delta=0.2)$ in case 1. The solid line on the proposed filter graphs presents the sign of Selector in (7).
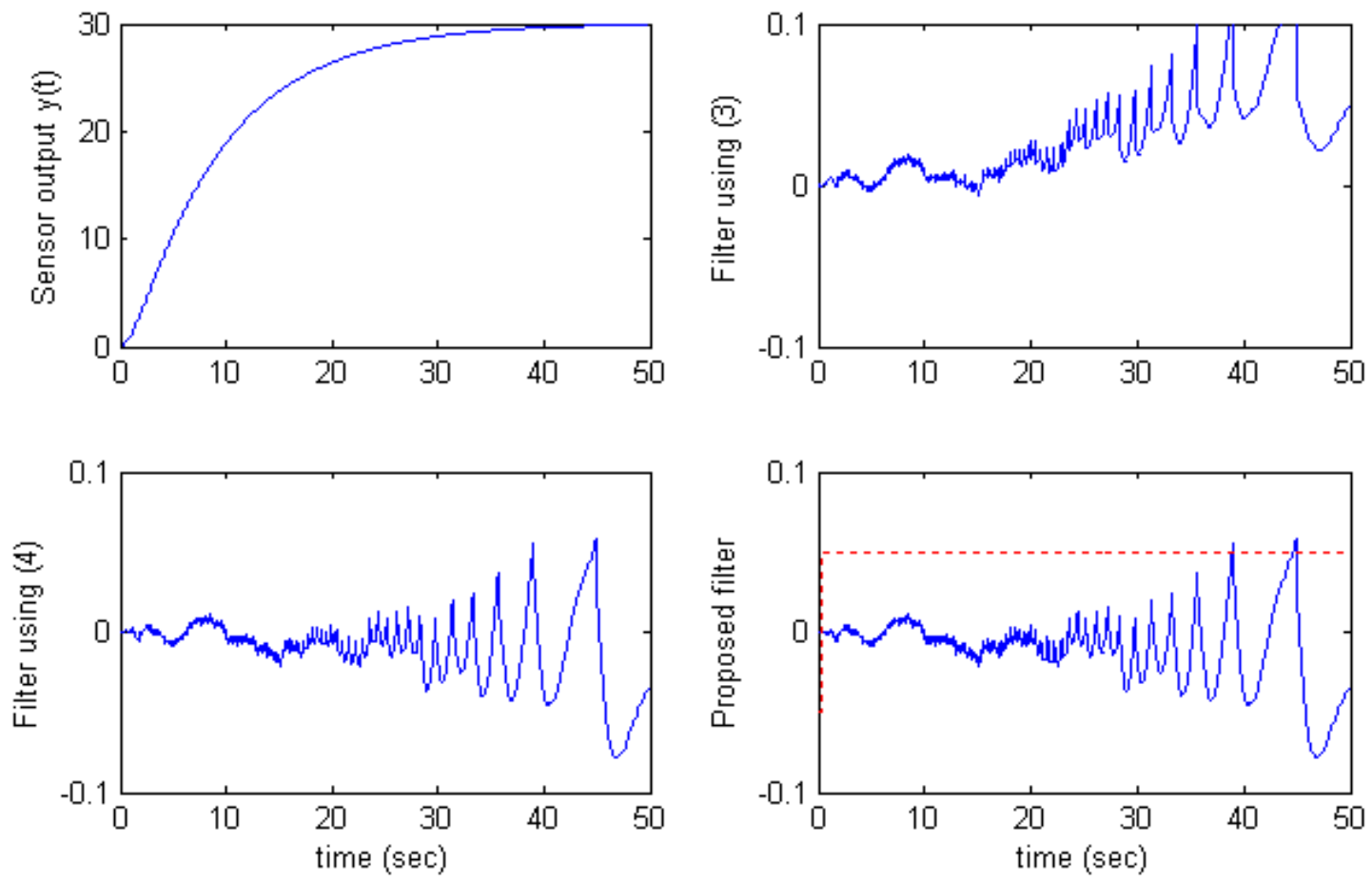

Figure 5. Estimation error of 3 filters $(\delta=0.2)$ in case 2.
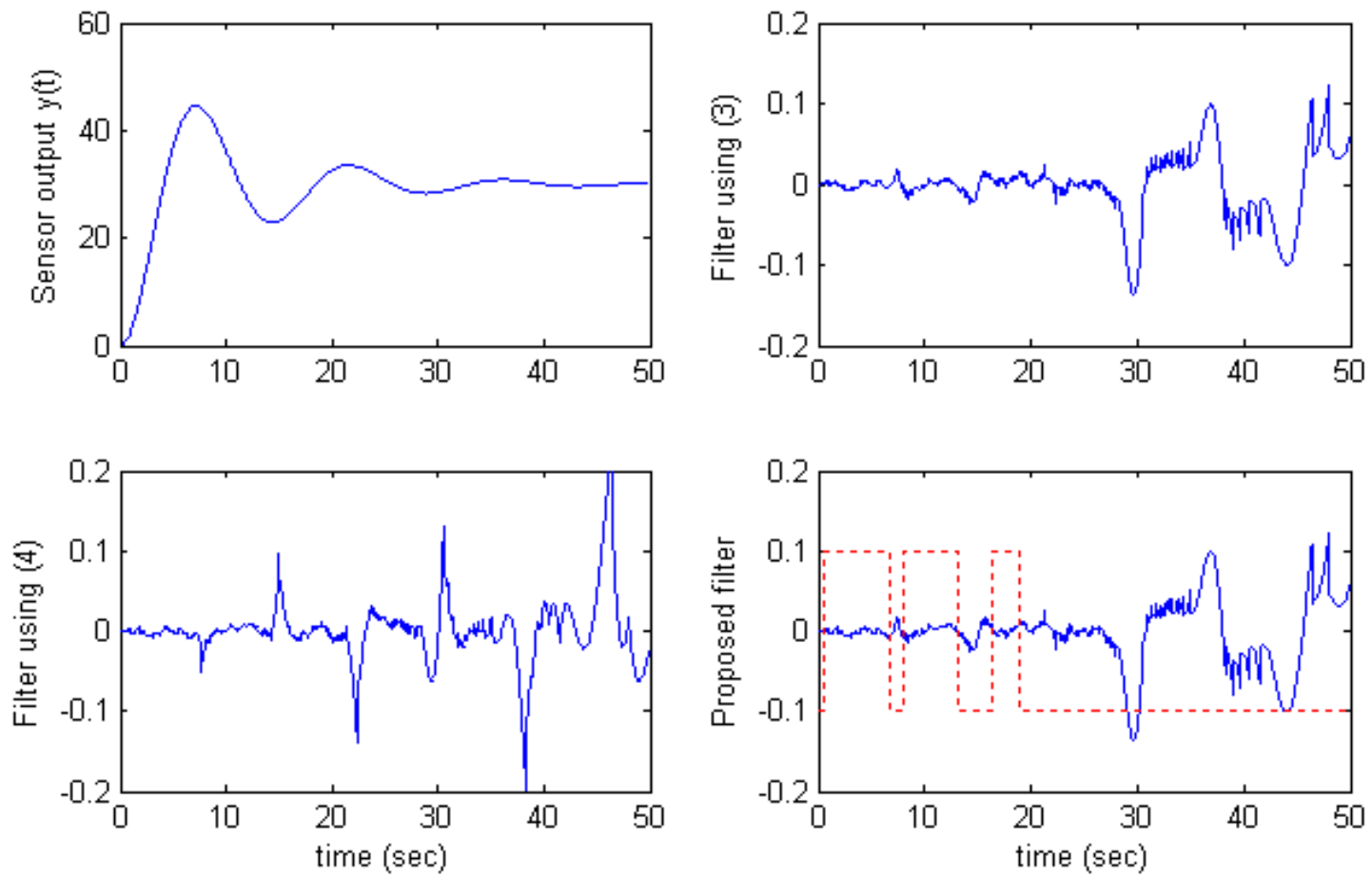
Figure 6. Estimation error of 3 filters $(\delta=0.2)$ in case 3 .
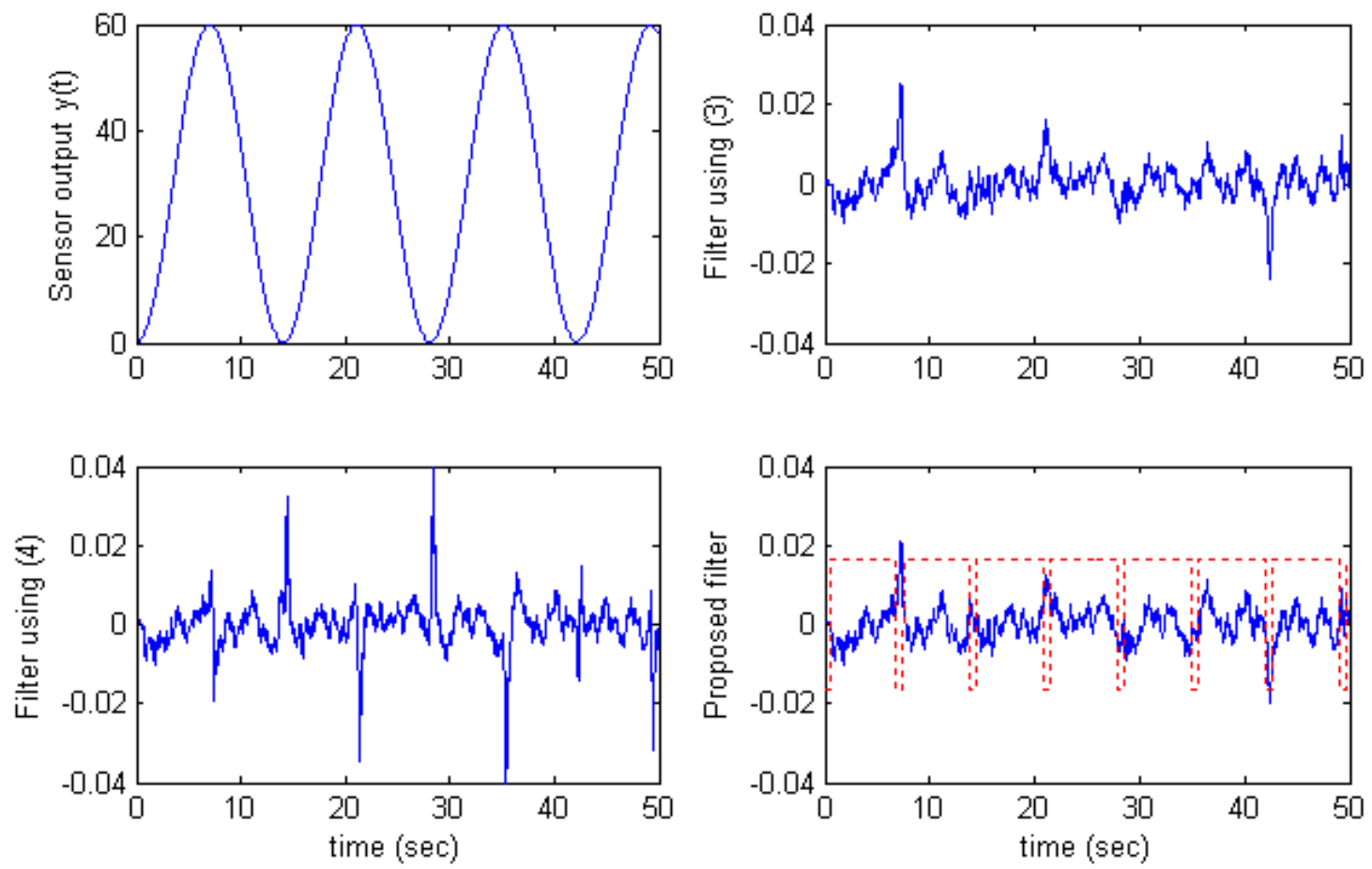

\section{Conclusion}

In this paper, the state estimation problem with SOD transmission method over the network has been considered. The main objective of this paper is how to reduce the estimation performance degradation when the SOD transmission method is used. With given $\delta$ value and measurement noise $R$, a suitable new noise is computed and compensated to the estimator so that estimation error is as small as possible. Accordingly, not only sensor data transmission rate can be significantly reduced, but also estimation performance degradation is relatively small. Through the simulations, it is shown that estimation performance of the proposed method is better than that of [5], where the total number of sensor data transmissions is identical.

\section{Acknowledgements}

This work was supported by the Korea Science and Engineering Foundation (KOSEF) grant funded by the Korea government(MOST) (No. R01-2006-000-11334-0). The authors also would like to thank financial support from post-BK(Brain Korea) 21 program. This work was supported by grant No. R012006-000-11334-0 from the Basic Research Program of the Korea Science \& Engineering Foundation.

\section{References and Notes}

1. Walsh, G. C.; Ye, H. Scheduling of networked control systems. IEEE Control Systems Magazine, 2001, 21(1), 57-65. 
2. Otanez, P. G.; Moyne, J. R.; Tilbury, D. M. Using deadbands to reduce communications in networked control systems. In Proceedings of the American Control Conference, Anchorage, U.S.A., 2002, 3015-3020.

3. Yook, J. K.; Tilbury, D. M.; and Soparkar, N. R. Trading computation for bandwidth: Reducing communication in distributed control systems using state estimators. IEEE Transactions on Control Systems Technology, 2002, 10(4), 503-518,.

4. Ishii, H.; Francis, B. A. Limited Data Rate in Control Systems with Networks. Nework: Springer, 2002.

5. Suh, Y. S.; Nguyen, V. H.; Ro, Y. S. Modified Kalman filter for networked monitoring systems employing a send-on-delta method. Automatica, 2007, 43(2), 332-338.

6. Miskowicz, M. Analytical approximation of the uniform magnitude-driven sampling effectiveness. Proceedings of IEEE International Symposium on Industrial Electronics ISIE'2004, Ajaccio, France, 2004, 407-410.

7. Mikowicz, M. Send-On-Delta Concept: An Event-Based Data Reporting Strategy. Sensors 2006, 65, 49-63.

8. Astrom, K. J.; Bernhardsson, B. M. Comparison of Riemann and Lebesgue sampling for first order stochastic systems. In Proceedings 41 st Conference on Decision and Control, Las Vegas, U.S.A., 2002, 2011-2016.

9. Plönnings, J.; Neugebauer, M.; Kabitzsch, K. A traffic model for networked devices in the building automation. In 2004 IEEE International Workshop on Factory Communication Systems, Vienna, Austria, 2004, 137-145.

10. Brown, R. G.; and Hwang, P. Y. C. Introduction to Random Signals and Applied Kalman Filtering, New York: John Wiley \& Sons, 1997.

11. Hernandez, W. Improving the response of several accelerometers used in a car under performances tests by using Kalman filtering. Sensors, 2001, 1, 38-52.

(C) 2007 by MDPI (http://www.mdpi.org). Reproduction is permitted for noncommercial purposes. 